unprotected home-based care of infected people and by modifying traditional burial practices. Infection-control measures protect healthcare workers. Together with rapid identification and isolation of ill people, and tracing and monitoring of their contacts for 21 days (the maximum incubation period of the disease), such measures have stopped Ebola outbreaks in the past.

But the dysfunctional health-care infrastructure of the three countries at the centre of the outbreak - Guinea, Sierra Leone and Liberia, which are poor and struggling to emerge from years of war - is simply not up to the task. The nations need help, and urgently.

The international community must mobilize now. Aid is increasing, but most of those involved, from governments and the World Health Organization (WHO) to researchers, all initially underestimated the threat. This is perhaps because most past outbreaks have been small and relatively straightforward to control.

The WHO has a part to play, but contrary to a widespread assumption, it does not have the in-house capacity to send large teams into the field. The agency's funding for outbreak responses has been slashed, and it has shifted focus to helping countries to reinforce their health systems so that they can respond better themselves. How the international community should best react to outbreaks, and what role the bureaucratic WHO should have, is a debate for after this outbreak is over. The pressing need now is to bring all available resources and talent to bear.

It is a sign of how desperate the situation has become that on 2 September, Joanne Liu, international president of medical group Médecins Sans Frontières (or Doctors Without Borders), called on countries to immediately deploy their military and civilian biodefence teams units that have been developed to respond to bioterror attacks. The crucial priorities, she said, are to scale up isolation centres, deploy mobile diagnostic labs (see page 145), build a network of field hospitals and establish dedicated air links to shift staff and equipment to where they are needed. In short, a military-style response, with its

associated strong chain of command, logistical capacities and speed. The concept makes a lot of sense and is an approach that governments should consider adopting - or explain why, if they choose not to do so. US President Barack Obama indicated last weekend that he would deploy the US military to assist in the outbreak.

It cannot be repeated enough that public-health measures and good old-fashioned epidemiological tracking of the infected and their con-

"The pressing need now is to bring all available resources and talent to bear."

tacts will bring this outbreak to an end. The priority must be to scale these up, alongside establishing more Ebola treatment centres on the ground. For instance, Ebola's high death rate could be slashed by better patient care, in particular by giving intravenous rehydration.

A highly effective Ebola vaccine would be a game-changer. A WHO-convened meeting on 4-5 September agreed on an unprecedented set of measures, including relaxing regulatory requirements so that experimental drugs and vaccines can be quickly tested under the difficult field conditions of this outbreak, and perhaps even widely deployed. The measures will, for example, permit expedited vaccine trials and informal clinical studies of drugs that could produce useful initial data within months.

Regulators and researchers should be applauded for their speed and pragmatism in exploring innovative methods for conducting trials during this outbreak. Crucially, all those who organize trials must be willing to standardize and share the data they collect to maximize their scientific and medical value, and to allow rapid decisions to be made on which products to prioritize.

West Africa's outbreak illustrates the serious weaknesses in the international community's ability to respond to outbreaks of emerging diseases, despite years of debate. It should also hammer home a truism for future planning - the costs of setting up infrastructure to ensure an early response are small compared with the huge social and economic costs of a large deadly disease outbreak.

\section{Orbital assembly}

\author{
The space launch of a 3D printer does not \\ herald a brave new era - but it is a good start.
}

$\mathrm{P}$ erhaps the most famous DIY job ever done in outer space was performed in April 1970 after an explosion disabled Apollo 13 on its way to the Moon. The three astronauts on board the craft scrambled together a makeshift adapter from cardboard, plastic bags and duct tape to scrub poisonous carbon dioxide from the air.

What if they had had access to a device that could design and manufacture replacement parts to order?

Last year, an engineer demonstrated just such a device: a threedimensional (3D) printer. Working for Made in Space in Moffett Field, California, the engineer spent an hour on the computer designing an adapter for the Apollo 13 scrubber, and the rest of the day printing it and demonstrating how it would work. Problem solved?

Perhaps it would be if every spacecraft had a $3 \mathrm{D}$ printer. Working with NASA, Made in Space is about to launch the first such printer into space (see page 156). If dreamers have their way, it will be the start of a new generation of manufacturing in orbit.

Imagine a rocket carrying little but a machine that can print the infrastructure for a colony on Mars. Or a spacecraft that can unfurl robotic tethers, printing and braiding giant ribbons into a starshade so that a telescope can stare, unblinded, at extraterrestrial worlds.

If this sounds impractical, it's because it is. For decades, enthusiasts have dreamed up ambitious ways to manufacture structures in space. A 1970s concept known as the beam builder would have welded

aluminium tubes together to create huge trusses spilling out of the back of the space shuttle. But in the 1990s, when countries began building hardware components for the International Space Station, they opted to do so in the familiar environment of planet Earth. Each large element was sent into space individually; only once aloft were the parts joined together to form the sprawling complex.

It would be wise to remember such lessons as the enthusiasm for 3D printing runs high. In July, a US National Research Council report concluded that there is a vast gap between what people think the technology can do and what it really can. It is all very well to pack a $3 \mathrm{D}$ printer for a journey to deep space - but what should a space traveller do when the printer itself breaks down? Carry a backup?

There is a place for $3 \mathrm{D}$ printing in space applications. Among other things, designers on the ground can dream up bizarre and fanciful parts, then print them regardless of many conventional design constraints. In principle, this means slimmer spacecraft that are cheaper to launch. That can be a big deal for an industry that must weigh every nut and bolt.

NASA is even talking about printing CubeSats, small box-shaped satellites that can be launched in flocks from a single launch vehicle or off the space station itself. A PrintSat, a CubeSat printed from a polyamide-based material, is slated for launch later this year as a test for how well such devices might survive in the harsh environment of space.

NASA is not alone. The European Space Agency is developing ways to use plastic and metal printed parts on the space station; the Italian Space Agency is hoping to send its own printer to the station in 2017.

D NATURE.COM To comment online, click on Editorials at: go.nature.com/xhunqv
Such experiments may not lead directly to a Martian base, but that is no reason not to encourage the fledgling technology. The maker ethos has permeated everywhere, it seems even beyond the gravitational pull of Earth. 